\title{
A Simple Method to Extract Whole Apolipoproteins for the Preparation of Discoidal Recombined High Density Lipoproteins as Bionic Nanocarriers for Drug Delivery
}

\author{
Wenli Zhang ${ }^{1}$, Ji Wang ${ }^{1}$, Junting Jia ${ }^{1}$, Liang Chen ${ }^{1}$, Zimei $\mathrm{Wu}^{2}$ and Jianping Liu ${ }^{1}$ \\ ${ }^{1}$ Department of Pharmaceutics, China Pharmaceutical University, Nanjing, PR China, ${ }^{2}$ School of Pharmacy, University \\ of Auckland, Private Bag, Auckland, New Zealand.
}

Received, March 20, 2015; Accepted, May 5, 2015; Published, May 9, 2015.

\begin{abstract}
Purpose: To develop a simple method to extract the whole apolipoproteins (apo) including apoA-I in native high density lipoproteins (HDLs) and prepare discoidal Tanshinone IIA-loaded reconstituted HDL (TA-rHDLs) as a dual functional drug delivery system with plaque-site target and therapeutic promises in atherosclerotic lesions. Methods: A method based on isoelectric precipitation coupled with organic solvent precipitation was developed to isolate the whole apolipoproteins (apos). TA-rHDLs were prepared by incubating the resultant apos with liposomes and the incubation conditions were optimized using fluorescence quenching experiment. TA-rHDLs were characterized in terms of size, zeta potential, morphology, interaction between lipid and apos, safety, and bionic function. Results: The extraction results showed that the yield of the HDL apos was $82.4 \%$, with $59 \%$ being apoA-I type, similar ratio of apoA-I in the native apos. TA-rHDL prepared were disc-like with an average diameter of $157.6 \pm 4.8$ $\mathrm{nm}$, zeta potential of $-20.90 \pm 0.15 \mathrm{mV}$, and entrapment efficiency of $(90.13 \pm 1.4) \%$. The interaction between the lipids and apos was electrostatic and hydrophobic force and was associated with amino acid sequence. Haemolysis and cytotoxicity assays showed good biocompatibility of TA-rHDL. Sterol efflux assay from macrophages mediated by TA-rHDLs and structure remodeling behavior from discs to spheres proved that TA-rHDL could resemble the biological activity of native nascent HDL irrespective of the size. Conclusions: The simple approach to isolate apos may provide a convenient and economical resource to support the development of rHDL as a potential targeting nanocarrier for lipophilic cardiovascular drugs.
\end{abstract}

This article is open to POST-PUBLICATION REVIEW. Registered readers (see "For Readers") may comment by clicking on ABSTRACT on the issue's contents page.

\section{INTRODUCTION}

It has been well established that high-density lipoproteins (HDLs) are the main actor in the process known as reverse cholesterol transportation (RCT), in which HDLs promote the transportation of excess cholesterol from extra-hepatic or peripheral tissue to the liver for elimination through the biliary system $(1,2)$. Natural HDLs, with a Stoke's diameter of 5-17 $\mathrm{nm}$ (3), exist in two different forms: one is nascent in discoidal shape, the other is matured in spherical shape. The discoidal HDLs consist of hydrophobic lipid bilayers (phospholipid and cholesterol) wrapped with apolipoproteins (apos). The spherical HDLs have a hydrophobic core of triglycerides and cholesterol esters coated with a monolayer of phospholipids in which apos are embedded. Nascent HDLs can display the role of RCT in which discoidal HDLs will receive cholesterol from cells and remodel their structure, yielding spherical HDL under lecithin:cholesterol acyl transferase (LCAT) which convert free cholesterol to cholesteryl ester (CE) (4). In the past decades, reconstituted HDLs (rHDLs) in nascent form have shown therapeutic promise for regression of atherosclerotic lesions $(5,6)$. Recently, rHDLs have been utilized as delivery carriers due to its attractive attributes $(7,8)$ such as capability (hydrophobic cores or phospholipids bilayers) for lipophilic drugs $(9,10)$, prolonged half-life and endogenesis. In our previous studies, reconstituted HDL nanocarriers $(11,12)$ in two forms have shown therapeutic effect of carriers and also

Corresponding Authors: Jianping Liu; Department of Pharmaceutics, China Pharmaceutical University, Nanjing, PR China; Email: jianpingliu1293@163.com. Zimei Wu; School of Pharmacy, University of Auckland, Auckland, New Zealand; Email: z.wu@auckland.ac.nz. 
target specificity towards the diseased vasculature in atherosclerotic rabbits (13).

Early studies have shown that isolated apoA-I could spontaneously interact with phosphatidylcholine at its phase transition temperature and form discoidal complexes (14), in the presence of the detergent sodium cholate. And the RCT role was reported to be mainly related to the phospholipid bilayer and apoA-I-mediated reaction (15). Hence, our hypothesis was that the size of discoidal rHDLs was not supposed to influence their structure and RCT effect as long as phospholipids bilayer and apoA-I were ensured. And more drugs would be loaded if the size of rHDLs was enlarged. Although apoA-I is the most abundant protein (roughly $70 \%$ of the HDL protein mass) (16) component of HDLs, other apos in HDLs such as apoA-II, apoE also play an essential role in HDL-therapy of coronary artery disease (17, 18). Therefore, the whole apos in HDLs were preferred to the single apoA-I to constitute the drug delivery system with carrier therapeutic effect. However, one of issues for rHDL preparation is the necessity to isolate apos as a starting material by consuming huge amount of human serum (19) or resort to cell expression systems that secrete recombinant human apos $(20,21)$. In the latter, the procedures to gain apos are very sophisticated in order to avoid immunological responses aroused by heterology.

In this paper, an economical and alternative method to obtain whole apos on HDLs from industrial waste during the production of albumin was introduced. The resultant product was used in the formulation of size-enlarged discoidal rHDLs using Tanshinone IIA (TA) as a model drug, a naturally occurring anti-atherosclerotic agent. To test the activity of extracted apos and prove the bionic function of size-enlarged rHDL, TA-rHDLs were prepared under mild conditions and tested by studying the sterol efflux mediated function and structure remolding behavior resembling native HDLs. The properties of TA-rHDLs such as morphology, entrapment efficiency (EE), size and distribution, interaction between apolipoproteins and lipid cores, biocompatibility were also characterized. The main contents of this work are presented graphically in Figure 1.

\section{Extraction of apos Discoidal TA-rHDLs Spherical TA-rHDLs}

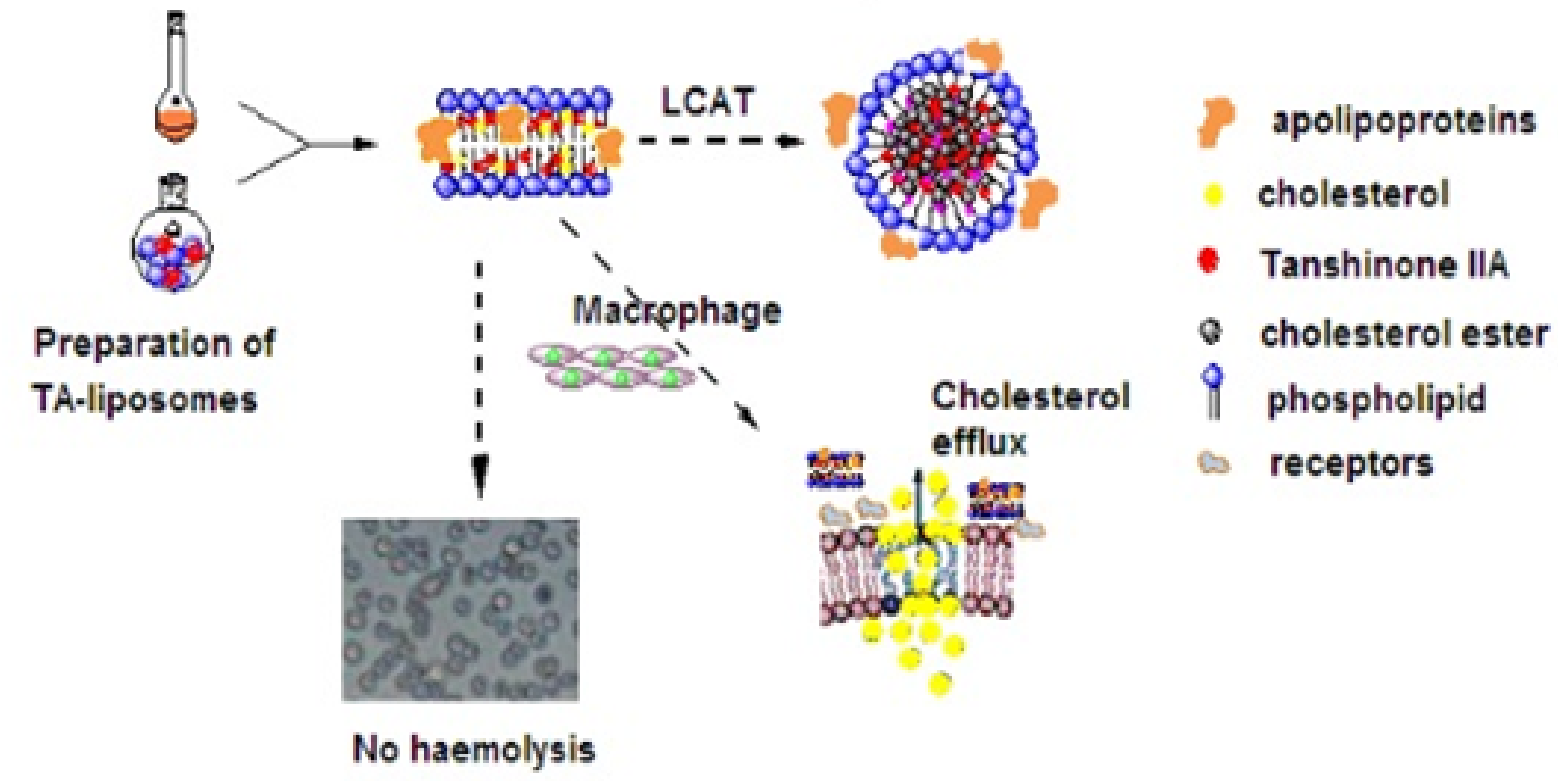

Figure 1. Schematic diagram of preparation procedure and bionic characterization for discoidal TA-rHDLs. 


\section{MATERIALS AND METHODS}

\section{Materials}

Tanshinone IIA (98\% pure) was purchased from Xi'an honson biotechnology Co., Ltd. (Xi'an, China). Fraction IV (FIV) precipitate (the raw material for extraction of apos) was kindly gifted by Tonrol Bio-Pharmaceutical Co., Ltd (Anhui, China). The precipitate is white powder and preserved at $-70{ }^{\circ} \mathrm{C}$ before use. Lipoid S 100 was obtained from Lipoid $\mathrm{GmbH}$ (Ludwigshafen, Germany). Egg lecithin PC-98T was supplied by Advanced Viecle Technology L.T.D. Co (Shanghai, China). Cholesterol was purchased from Sigma-Aldrich Co., Ltd (Shanghai, China). Sephadex G50 was purchased from Pharmacia (Uppsala, Sweden). RAW 264.7 cells were obtained from American Type Culture Collection. Native HDLs were prepared by two-step ultracentrifuge in our laboratory (22). All other reagents used in this study were of analytical grade except methanol of chromatographic grade.

\section{Extraction of apolipoproteins in HDLs}

A so called FIV precipitate, generated as waste during industrial production of albumin in human serum, was employed to extract apos in HDLs. Approximately, $50 \%$ of in vivo apoA-I was retained in FIV precipitate.

In our study, a novel method based on isoelectric precipitation coupled with organic solvent precipitation method was adopted to isolate whole apos in native HDLs from the FIV precipitate: Firstly, $15 \mathrm{~g}$ FIV was suspended in 150 $\mathrm{ml}$ phosphate buffer saline (PBS, $0.02 \mathrm{M}, \mathrm{pH}=5.5$ ) allowing sufficient dissolution of the soluble impurities. Anhydrous ethanol was added (final concentration $20 \%$ ), and the resulted suspension was centrifuged at $13000 \mathrm{r} / \mathrm{min}$ and $4{ }^{\circ} \mathrm{C}$ for 20 min (Hitachi Himac CR 21G, Tokyo, Japan). The sediment was collected and resuspended in $100 \mathrm{ml}$ PBS (0.05 M, pH=6.6), then $10 \mathrm{ml}$ anhydrous ethanol was added and a second centrifugation step was performed with the same condition. The supernatant was pooled and filtered through $0.8 \mu \mathrm{m}$ filters. The filtrate was adjusted to $\mathrm{pH}=5.5$ using PBS (1.65 M, pH=3.6) containing $18 \%$ ethanol and centrifuged with the same condition to get the sediment, which was expected to be HDLs.

To separate the apos from the lipids in the HDLs, the HDLs were suspended in $1 \mathrm{ml}$ Tris- $\mathrm{HCl}$ buffer (0.01 M, pH 8.0) and added dropwise into 50 $\mathrm{ml}$ ethyl ether: ethanol (2:3) allowing delipidation for $12 \mathrm{~h}$. The apos were obtained from the precipitate after centrifuge. The solid apos were obtained after lyophilization and stored at $-70{ }^{\circ} \mathrm{C}$ until use. FIV was also delipidated with the same procedure in order to estimate the extraction yield.

\section{Verification of the extracted apolipoproteins}

To ensure the apos extracted were free of lipid and to determine their composition, the delipidation efficiency was determined by Bradford method (23). The extracted HDLs before delipidation were stained by Sudan Black and determined by agarose gel electrophoresis as described previously (12), and the delipidated FIV precipitate and the extracted apos were both applied for sodium dodecyl sulfate polyacrylamide gel electrophoresis (SDS-PAGE) as described previously (12). After completion of electrophoresis, the gels were photographed with gel image system (GelDoc 2000, Bio-Rad, Hercules CA, USA). The molecular weight of protein was calculated by linear regression between migrated distance and logarithm of molecular weight.

Western blotting was employed to confirm the extracted apos containing apoA-I. After being separated by SDS-PAGE, apoA-I was transferred to nitrocellulose membranes $\left(100 \mathrm{~V}, 4{ }^{\circ} \mathrm{C}, 90 \mathrm{~min}\right)$ and detected with a polyclonal goat anti-human apoA-I antibody (Abcam, cambridge, UK), followed by a horseradish peroxidase-conjugated donkey anti-goat IgG (Santa Cruz, CA, USA). Detection was performed using the enhanced chemiluminescence (ECL) method.

The concentrations of proteins in delipidated FIV precipitate and extracted apos were determined using Coomassie kits (Jiancheng Bioengineering Institute, Nanjing, China) based on the protocol provided. Densities and areas of band signals on SDS-PAGE gels were quantified using software Quantity One (version 4.0, Bio-Rad) to determine the percentage $\left(D_{l}\right)$ of apoA-I in the extracted apos or the percentage $\left(D_{0}\right)$ of apoA-I in the defatted FIV. By the mean of apoA-I, the extraction yield of apos $(P)$ was calculated based on the following formula:

$$
P(\%)=\frac{M_{\text {apo }} \times D_{1}}{M_{\text {pro }} \times D_{0}} \times 100
$$

Where $M_{\text {apo }}$ is the average mass of extracted apos from each batch and $M_{\text {pro }}$ is the average mass 
of total protein in the defatted FIV precipitate from each batch.

\section{Preparation of TA-rHDLs}

Two procedures were implemented to prepare TA-rHDL discs: construction of the TA liposomes (named as TA-rHDL cores) and subsequent incubation with apolipoproteins.

The TA liposomes were prepared by a thin-film dispersion method. Briefly, TA, lecithin and cholesterol (weight ratio $=0.5: 3: 6$ ) were dissolved in dehydrated alcohol and dried in an eggplant-shaped flask. Tris- $\mathrm{HCl}$ buffer $(15 \mathrm{ml}, 0.1 \mathrm{M} \mathrm{KC1}, 1 \mathrm{mM}$ EDTA, $\mathrm{pH}$ 8.0) with or without sodium cholate was added to hydrate the thin-film, then the mixture was vortexed thoroughly for $5 \mathrm{~min}$, followed by ultrasonication for $300 \mathrm{~s}$ using an Ultrahomogenizer (JY 92II, Ningbo, China) until a clear suspension was obtained. The TA liposomes were then obtained by filtering through $0.22 \mu \mathrm{m}$ filters to remove larger particles.

The TA-rHDL discs were prepared by the sodium cholate reconstitution method (14). The TA liposomes obtained above were incubated with the extracted apos with or without sodium cholate (Sodium cholate could be added either in the hydration medium or in the preformed TA liposomes suspension). Fluorescence quenching experiment was performed to determine the optimal conditions in order to achieve the highest apos coupling efficiency for TA-rHDLs. In the last, TA-rHDLs were dialyzed to remove sodium cholate using dialysis bags (MW cut off 14,000 Da) against $0.15 \mathrm{M} \mathrm{NaCl}, 1 \mathrm{mM}$ sodium EDTA, and $0.01 \mathrm{M}$ Tris- $\mathrm{HCl}$ buffer.

\section{Formulation and technique optimization of TA-rHDLs}

The intrinsic fluorescence quenching experiments can reveal the interaction of tryptophan residues in apos with lipid cores, which reflects the apos coupling efficiency with lipids in TA-rHDLs. Fluorescence spectra and integrated intensities were recorded with a Shimadzu RF-5301PC fluorescence spectrophotometer using $282 \mathrm{~nm}$ exciting light and $5 \mathrm{~nm}$ excitation and emission slit widths. Specifically, spectra were recorded at $25{ }^{\circ} \mathrm{C}$ by adding same concentration of TA-rHDLs into KI with concentrations ranging from 0 to $0.3 \mathrm{M}$. The $2.5 \mathrm{M}$ KI stock solutions contained $1 \mathrm{mM} \mathrm{Na} 2 \mathrm{~S}_{2} \mathrm{O}_{4}$ in order to prevent $\mathrm{I}_{3}^{-}$ formation. The results were analyzed by the Stern-Volmer equation as modified by Lehrer (24):

$$
\frac{F_{0}}{\Delta F}=\frac{1}{f_{a}}+\frac{1}{f_{a} K_{s}[K I]}
$$

where $F_{0}$ is the initial integrated fluorescence intensity, $\Delta F$ is the difference between $F_{0}$ and the observed fluorescence intensity at each KI concentration, $f_{a}$ is the fraction of exposed tryptophan residues, and $K_{s}$ is the Stern-Volmer constant.

The percentage of apos anchoring to the TA-rHDL cores were estimated through $f_{a}$ which can be calculated from the y-axis intercept of the linear plot of $F_{0} / \Delta F$ against $1 /[\mathrm{KI}]$. Therefore, a low $f_{a}$ value would indicate low ratio of exposed tryptophan residues in TA-rHDLs, which reflects high coupling efficiency between the apos and lipids in TA-rHDLs. Different types of lecithin from soybean and egg, quantity of sodium cholate and apolipoprotein, order of adding sodium cholate during or before incubation with apos, incubation duration period were screened by comparing apolipoprotein coupling efficiency. Specifically, apos powder were dissolved in TA-rHDL core suspensions, then stirred $(600 \mathrm{rpm})$ at $4{ }^{\circ} \mathrm{C}$ (The formulation design was listed in Table 1). Then the fluorescence quenching experiments described above were carried out to calculate exposure ratio of tryptophan residues $f_{a}$. The optimal preparation conditions would be determined by the lowest $f_{a}$.

\section{Interaction of apolipoproteins with lipid cores}

To investigate the binding interaction between the lipid cores and apos, neutral (without sodium cholate), negative-charged (sodium cholate was added in hydration medium before incubation with apos) and positive-charged lipid particles (octadecylamine was added with phospholipid before incubation with apos) were prepared to incubate with apos. Denatured apos with altered space conformation obtained by heating at $95^{\circ} \mathrm{C}$ were also employed to explore the influence of protein conformation and amino acid sequences on binding force. After incubating different TA-rHDL cores (TA liposomes) with apos denatured or not, intrinsic fluorescence quenching experiments were undertaken to determine the binding force by comparing the exposure ratio of tryptophan residues $f_{a}$.

Size, zeta potential and entrapment efficiency Size and zeta potential of TA-rHDLs were measured with dynamic light scattering (DLS) 
using a Zetasizer 3000 HSA (Malvern, U.K.). Samples were diluted appropriately with aqueous phase prior to the measurements.

Drug entrapment efficiency was determined using the mini-column centrifugation method (25). The concentrations of TA entrapped in the TA-rHDLs $(C)$ and the total drug in TA-rHDLs suspension $\left(C_{0}\right)$ were determined by HPLC, respectively, after dilution with absolute ethanol. Entrapment efficiency was calculated with the following formula:

$$
E E(\%)=\frac{C}{C_{0}} \times 100
$$

\section{Visualization by transmission electron microscopy (TEM)}

The microstructures of rHDLs before and after incubation with apos were all examined by TEM (H-7650, Hitachi, Japan) with negative stain method. Samples were diluted appropriately with aqueous phase before preparation for TEM. A drop of each sample was applied to a copper grid coated with carbon film and air-dried; 2\% (w/v) phosphotungstic acid (PTA) solution was then dropped onto the grids. After being negatively stained and air-dried at room temperature, the samples were accomplished for the TEM investigation.

\section{Haemolysis tests}

Fresh rabbit blood was centrifuged at $1500 \mathrm{rpm}$ for 15 min. The supernatant was removed and the resultant concentrated red blood cells (RBCs) were washed with $0.9 \%$ sodium chloride solution. This process was repeated twice and RBCs were resuspended with normal saline to an approximate concentration of $2 \%(\mathrm{v} / \mathrm{v})$. TA-rHDLs, their lipid cores (TA liposomes) and blank rHDLs all before and after dialysis, normal saline (as negative control) and distilled water (as positive control) were mixed with a suspension $(2 \%, \mathrm{w}: \mathrm{v})$ of fresh rabbit red blood cells. TA formulations were diluted by normal saline with the dilution factors of $5,6.25,8.3,12.5$, and 25 to obtain different drug concentrations of $1.2,1.5,2,3$ and $6 \mu \mathrm{g} / \mathrm{ml}$. Drug loading was all kept at $4.6 \%$, therefore, the lipid concentrations were $24.9,31.1,41.5,62.2,124.4$ $\mu \mathrm{g} / \mathrm{ml}$, respectively. The blank HDLs were also diluted to the same lipid concentrations. After incubation at $37{ }^{\circ} \mathrm{C}$ for $3 \mathrm{~h}$, the sample was centrifuged at $1500 \mathrm{rpm}$ for $15 \mathrm{~min}$. The absorbance of released hemoglobin in the supernatant for different samples was measured by UV spectrophotometer at $577 \mathrm{~nm}$ and the morphology of incubated red blood cells was visualized under a microscope (Nikon eclipse Ti-S, Japan). The absorbance of positive control was set as $100 \%$ haemolysis while that of negative control indicates $0 \%$ haemolysis. The following equation was used in order to determine the percentage of haemolysis:

\section{Structure remodeling of TA-rHDLs under the action of $L C A T$}

Since the cholesterol was converted to ester during structure remodeling, cholesterol residue after reaction under LCAT could be used to evaluate the structure remolding degree of TA-rHDLs. LCAT were extracted by the method reported by Matz and Jonas (26). The average specific activity was assayed with Genmed LCAT kit (Genmed scientifics U.S.A) around $38 \mathrm{nmol} \mathrm{CE} / \mathrm{min} / \mathrm{ml}$. LCAT were added in $100 \mu \mathrm{L}$ blank rHDLs and 100 $\mu 1$ mercaptoethanol in pre-equilibrium buffer and incubated on a shaking-bed (SHZ-82A, Jintan, China) under $120 \mathrm{rpm}$ at $37^{\circ} \mathrm{C}$ for $10 \mathrm{~min}, 20 \mathrm{~min}$, $30 \mathrm{~min}, 45 \mathrm{~min}, 60 \mathrm{~min}$, respectively. After that, the reaction was stopped by adding cyclohexane to extract cholesterol from incubation solution. Then the cholesterol in cyclohexane was determined by pre-column derivation method as described above.

Blank rHDLs and TA-rHDLs with the same size and different drug-loading levels $(0 \%, 1.2 \%$, $3.1 \%, 4.6 \%$ ) were prepared and employed to investigate the effect of drug loadings on structure remodeling. Then the remolding reaction was conducted under the same condition and cholesterol residue was measured as described above.

The structure remolding behavior of TA-rHDLs was also observed by TEM. TA-rHDLs $(100 \mu \mathrm{l})$ were incubated with LCAT in $100 \mu 1$ mercaptoethanol in pre-equilibrium buffer and incubated on a shaking-bed under $120 \mathrm{rpm}$ at $37^{\circ} \mathrm{C}$. Then the enzymatic reaction was stopped after optimum duration by adding vitamin $\mathrm{C}$ before observation. Then the TA-rHDLs were taken to prepared transmission electron microscopy (TEM) samples as routine procedure. 


$$
\text { Haemolysis }(\%)=\frac{A b s_{\text {sample }}-A b s_{\text {negative control }}}{A b s_{\text {positive control }}-A b s_{\text {negative control }}} \times 100
$$

\section{STATISTICAL ANALYSIS}

Data reported were arithmetic mean values \pm standard deviation. Statistically significant differences were determined using two-tailed Student's t-test with $p<0.05$ as a level of significance.

\section{RESULTS AND DISCUSSION}

\section{Identification of extracted apos}

Native HDLs and extracted HDLs presented one same band in agarose gel electrophoretic pattern (Figure 2 A), which indicated high purity of isolated HDLs without other lipoproteins. The apos delipidated from isolated HDLs were white powder with a protein rate of $96.81 \%$ determined by Coomassie kit, indicating efficient delipidation. SDS-PAGE (Figure 2 B) for both extracted apos and proteins in FIV precipitate showed a major band at the molecular weight (MW) of $28.7 \mathrm{KDa}$, which is corresponding to the molecular weight of apoA-I. This was further confirmed by positive western blot assay (Figure $2 \mathrm{C}$ ). The percentage of apoA-I in the total proteins of FIV and the extracted apos determined by Quantity One software were $19.3 \%$ and $58.8 \%$, respectively. The increased ratio of apoA-I suggested that the apos belonging to HDL were refined from FIV precipitate. The ratio of apoA-I in the extracted apos was similar to that reported in the native apos of HDLs $(58.8 \%$ v.s.$$
\text { A }
$$
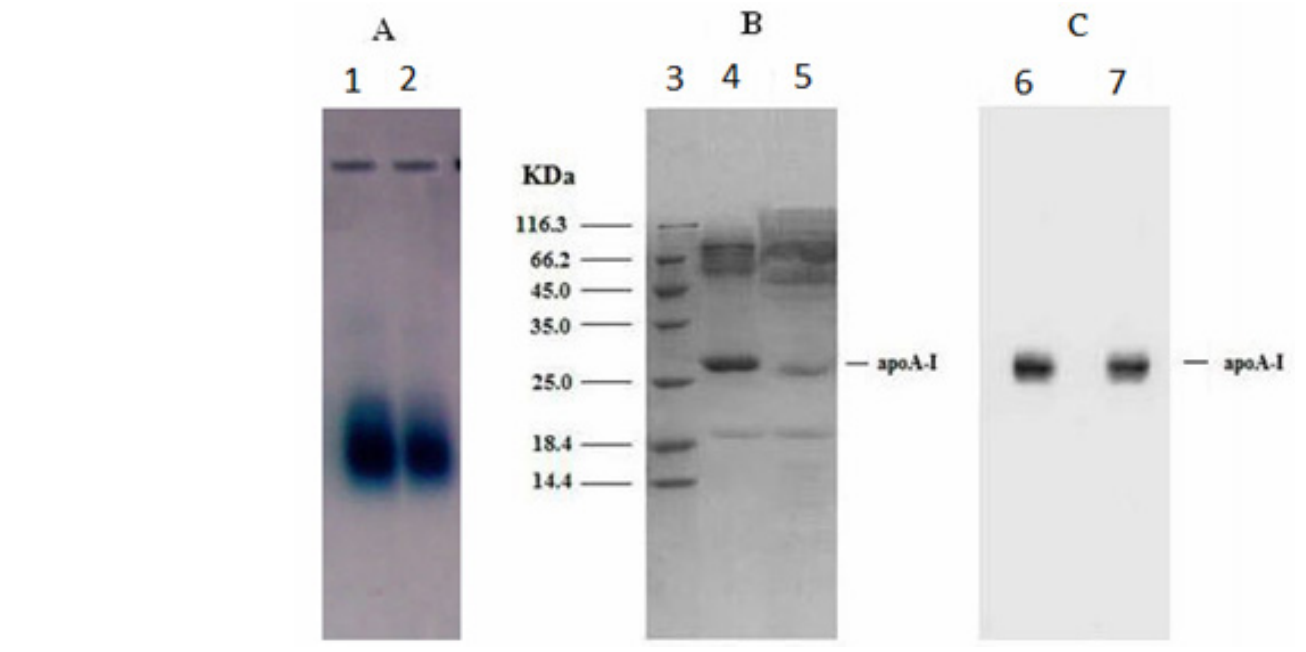

70\%). Given $85 \mathrm{mg}$ of apos was obtained from 15 g FIV precipitate, the yield of apoA-I, therefore, was calculated according to equation (1) as $82.4 \%$.

\section{Preparation of TA-rHDLs}

Lipid cores (TA liposomes) were more stable and little drug leaked out at low temperature in our preliminary experiments. In addition, sonication method which could facilitate apo binding with lipids might result in denaturation of apos due to the cavitation stress it involved for local temperature extremes (27) and free radical formation (28). To ensure the activity of apos and high EE of TA-rHDL, a mild rate of $600 \mathrm{rpm}$ was employed at $4{ }^{\circ} \mathrm{C}$ alternatively for all the following formulation studies.

Judging from the results of fluorescence quenching experiments as shown in Table 1, incubation time had no effect on apo coupling efficiency evidenced by no significant $f_{a}$ between formulation No. 1, 9, and 10. Therefore, $4 \mathrm{~h}$ was chosen as optimal incubation period. Although the coupling efficiency was slightly smaller when apos used were $14 \mathrm{mg}$ compared to $7 \mathrm{mg}$, the apo coupling quantity (total mass of apos $\times$ apo coupling efficiency) was the most (Formulation No. $1,7,8)$, so $14 \mathrm{mg}$ of apos was utilized. Lecithin (Formulation No. 2 v.s. 1), order of adding sodium cholate (Formulation No. 3 v.s. 1) and quantity of sodium cholate (Formulation No. 4, 5, 6 v.s. 1) all significantly affected apo coupling efficiency.

Figure 2. Agarose gel (A), SDS-PAGE (B) and Western blotting (C) electrophoretic patterns for different samples. 1: native HDLs, 2: extracted HDLs, 3: molecular weight standards, 4: apos in the extracted HDLs, 5: apos in the FIV precipitate, 6: apoA-I in native HDLs, 7: apoA-I in the extracted HDLs. 
Ultimately, the optimal conditions (Formulation No. 7) were determined with the highest apo coupling quantity for TA-rHDLs: $5 \mathrm{mg}$ sodium cholate was added in the water phase during lipid potion preparation (before incubation) and $2 \mathrm{ml}$ of TA-rHDL cores (TA liposomes) prepared with soybean lecithin were incubated with $14 \mathrm{mg}$ apos under $600 \mathrm{rpm}$ stirring at $4{ }^{\circ} \mathrm{C}$ for $4 \mathrm{~h}$.

\section{Characterization of TA-rHDLs}

The population of TA-rHDLs cores (TA liposomes) was relatively more homogeneous in size, with poly distribution index (PDI) increased after incubation with apos. As expected, the coupling of apos influenced the zeta potential values. The increased absolute value of zeta potential in TA-rHDLs after incubation further proved the incorporation of the amphipathic apos, which was negative charged in Tris-HCl buffer $(\mathrm{pH}=8.0)$. The increased size was also confirmed by TEM, which indicated that TA-rHDLs might form a complex of lipid cores coated with apos. TA-rHDL cores (TA liposomes) (Figure 3 A) were demonstrated liposomes but changed to heterogeneous discoidal stacks and stacked together (26) after incubation with apos (Figure $3 \mathrm{~B}$ ), which offered an explanation for the particle size heterogeneity. It has been proposed that apos would adopt a helical "hairpin" model which make liposomes discoidal (29) after binding with liposomes. The structural change after incubation from round vesicles to discoidal stacks may be the main reason for decreased EE after incubation and were same with our previous study (13).

Table 1. Fluorescence quenching results of TA-rHDL prepared with different formulae and technique (mean $\pm S D, n=3)$.

\begin{tabular}{|c|c|c|c|c|c|c|}
\hline No. & type of lecithin & $\begin{array}{l}\text { Order of adding } \\
\text { sodium cholate }\end{array}$ & $\begin{array}{c}\text { Quantity of } \\
\text { sodium cholate } \\
\text { (mg) }\end{array}$ & $\begin{array}{c}\text { Quantity of } \\
\text { apolipoproteins } \\
\text { (mg) }\end{array}$ & $\begin{array}{l}\text { Incubation } \\
\text { period (h) }\end{array}$ & $f_{a}$ \\
\hline 1 & Soy lecithin & $\begin{array}{l}\text { Added before } \\
\text { incubation }\end{array}$ & 5 & 7 & 4 & $0.264 \pm 0.01$ \\
\hline 2 & Egg lecithin* & $\begin{array}{l}\text { Added before } \\
\text { incubation }\end{array}$ & 5 & 7 & 4 & $0.380 \pm 0.01$ \\
\hline 3 & Soy lecithin & $\begin{array}{l}\text { Added during } \\
\text { incubation* }\end{array}$ & 5 & 7 & 4 & $0.502 \pm 0.02$ \\
\hline 4 & Soy lecithin & $\begin{array}{l}\text { Added before } \\
\text { incubation }\end{array}$ & $2.5^{*}$ & 7 & 4 & $0.318 \pm 0.01$ \\
\hline 5 & Soy lecithin & $\begin{array}{l}\text { Added before } \\
\text { incubation }\end{array}$ & $10^{*}$ & 7 & 4 & $0.428 \pm 0.03$ \\
\hline 6 & Soy lecithin & $\begin{array}{l}\text { Added before } \\
\text { incubation }\end{array}$ & $20 *$ & 7 & 4 & $0.422 \pm 0.03$ \\
\hline 7 & Soy lecithin & $\begin{array}{l}\text { Added before } \\
\text { incubation }\end{array}$ & 5 & 14 & 4 & $0.297 \pm 0.01$ \\
\hline 8 & Soy lecithin & $\begin{array}{l}\text { Added before } \\
\text { incubation }\end{array}$ & 5 & $21^{*}$ & 4 & $0.546 \pm 0.01$ \\
\hline 9 & Soy lecithin & $\begin{array}{l}\text { Added before } \\
\text { incubation }\end{array}$ & 5 & 7 & 8 & $0.267 \pm 0.01$ \\
\hline 10 & Soy lecithin & $\begin{array}{l}\text { Added before } \\
\text { incubation }\end{array}$ & 5 & 7 & 12 & $0.268 \pm 0.01$ \\
\hline
\end{tabular}


Table 2. Size, PDI, zeta potential, and EE of different TA formulations (means $\pm S D, n=3$ ).

\begin{tabular}{lcccc}
\hline Formulations & Size $(\mathbf{n m})$ & PDI & Zeta potential $(\mathbf{m V})$ & EE (\%) \\
\hline TA liposomes & $51.9 \pm 2.3$ & $0.132 \pm 0.017$ & $-0.31 \pm 0.04$ & $98.25 \pm 1.1$ \\
TA-rHDLs & $157.6 \pm 4.8^{*}$ & $0.347 \pm 0.015$ & $-20.90 \pm 0.15^{*}$ & $90.13 \pm 1.4$ \\
\hline
\end{tabular}

*means significant differences vs TA liposomes.
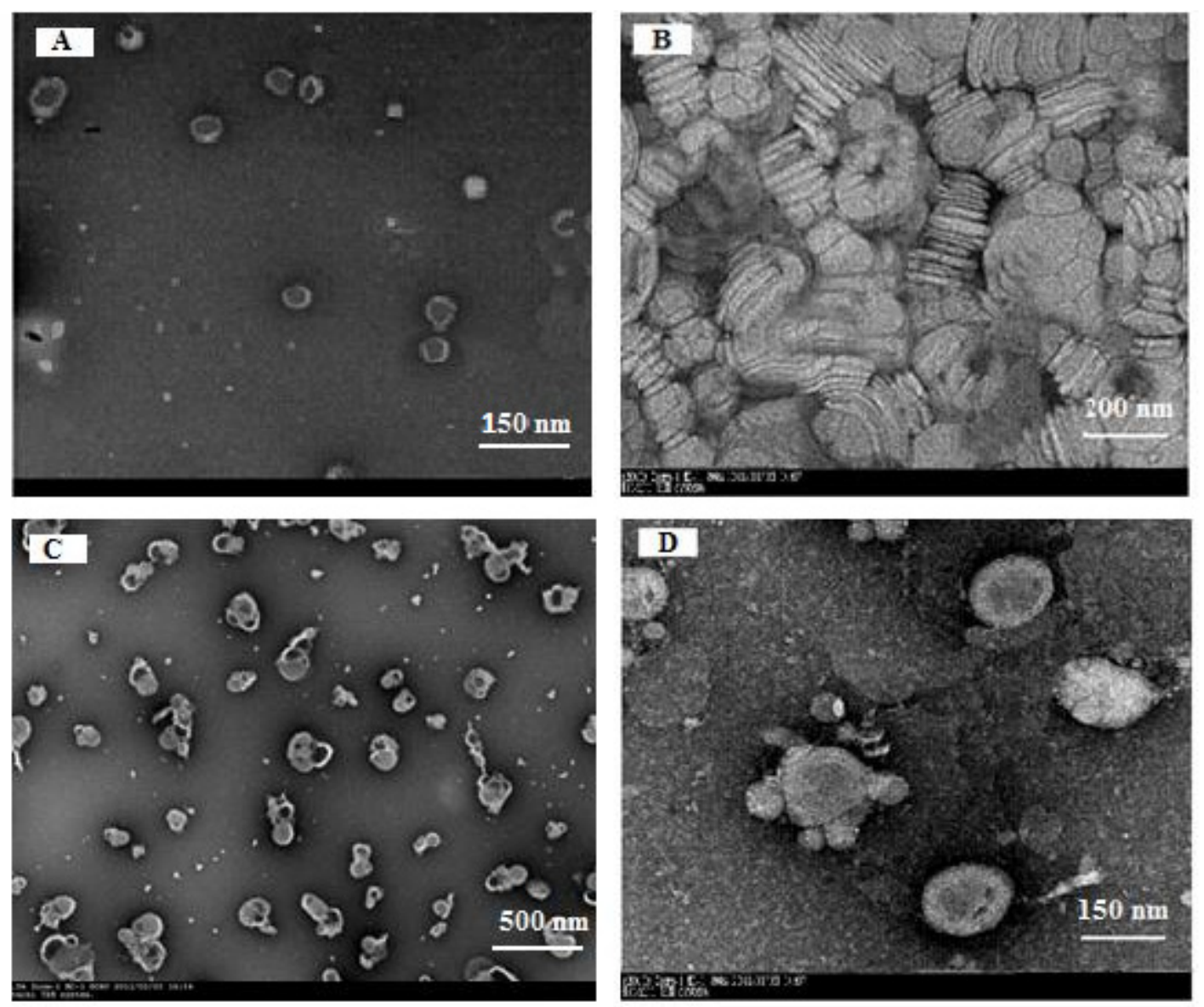

Figure 3. Representative TEM photographs of TA-rHDL cores (A), TA-rHDLs (B) and TA-rHDLs after incubation with LCAT in $100 \mu \mathrm{L}$ mercaptoethanol in pre-equilibrium buffer for $30 \mathrm{~min}$ at $37^{\circ} \mathrm{C}(\mathrm{C}$ and $\mathrm{D})$. D is higher magnification for $\mathrm{C}$.

\section{Interaction of apolipoproteins with lipid cores}

The $f_{\mathrm{a}}$ values (fraction of exposed tryptophan residues) of the fluorescence quenching experiments (Table 2) suggested that an increased ratio (about 1/4) of the fluorophores from 0.744 to 0.966 in the free apos after denaturation were accessible to be quenched by KI. After denaturation, all the tryptophan residues in the sequence of
apoA-I were exposed without spatial hindrance and could be quenched by KI. If the contributions to the overall fluorescence by all four tryptophan residues in apoA-I are similar, then only one tryptophan residue (1/4) appears to be inaccessible in spatial structure to the environment. This suggested the apos used for reconstitution of TA-rHDLs had the similar ratio of Trp residues to that of the 
commercial pure apoA-I (30). Although the apos used in this study were mixture in which apoA-I accounted for $\sim 60 \%$, other kinds of apo seemed not to contribute to $f_{\mathrm{a}}$ value. Therefore, the fluorescence quenching method could apply to study the binding of the whole apos in the HDL with lipid cores.

Generally, the interactions between the apos and lipid cores in the native HDLs are considered to be non-covalent binding $(29,31)$. The interaction of apos with lipid cores could be investigated by the decreased $f_{\mathrm{a}}$ (fraction of exposed tryptophan residues) in the TA-rHDLs since tryptophan residues of apos interacted with the lipid cores could not be quenched by KI. It was found that the negatively charged lipid cores prepared with sodium cholate had higher binding with apos than those prepared with octadecylamine or no surfactant. This was possibly due to the stronger electrostatic interaction between lipid bilayers and the positively charged residues of apos in apoA-I. The differences in affinity were not simply related to the distribution of charge residues along the axis of the helix of the apos but also related to their hydrophobic moment (31). A lower $f_{\mathrm{a}}$ for neutral rHDLs compared to free apos proved this point. It was reported (29) that the amphipathic $\alpha$-helices separated by Pro residues allow the interaction of the protein with phospholipids through its hydrophobic face, while the hydrophilic face of the helices interacts with aqueous phase. In addition, TA-rHDLs prepared with denatured apos had less accessible tryptophan residues to quench than denatured apos in free form, suggesting that its lipid-binding was more associated with amino acid sequence than spatial conformation.

\section{Haemolysis tests}

As shown in Figure 4, despite the same concentration of drug in TA-rHDLs and their cores (TA liposomes), their haemolysis effect was quite different. It was found that TA-rHDLs with apos, irrespective of dialysis, only caused less than 5\% haemolysis at all concentrations. Moreover, the red blood cells manifested intact and biconcave shape visualized by a red point in the middle of cells (Figure $5 \mathrm{E}, \mathrm{F}$ ), except that their aggregation status was a little different. The aggregation of red cells may be induced by sodium cholate or exposed apos on TA-rHDLs before dialysis. However, TA-rHDL cores (TA liposomes) could lead to $16 \%$ haemolysis even at the lowest concentration and the haemolytic effect was concentration-dependent. Their haemolysis toxicity aggravated after dialysis, which could be explained by the possible drug leakage from TA-rHDL cores (TA liposomes) during dialysis. Inferred from nonhaemolytic drug free rHDLs irrespective of dialysis (Figure 5 C, D) and the drug concentration-dependent haemolytic toxicity, the drug exposure on the membrane of TA-rHDL cores (TA liposomes) should be responsible for the haemolysis. TA-rHDLs formed a structure (11) of TA-rHDL cores surrounded by amphiphilic apos which can prevent drug leakage and isolate drug from interaction with red blood cells.

Table 3. Fluorescence quenching results of apolipoproteins by KI in different forms and binding with different kinds of TA-rHDL cores (mean $\pm \mathrm{SD}, \mathrm{n}=3$ ).

\begin{tabular}{lc}
\hline \multicolumn{1}{c}{ Sample } & $\boldsymbol{f}_{\boldsymbol{a}}$ \\
\hline Apoliporoteins (free form) & $0.744 \pm 0.006$ \\
Denatured apos (free form) & $0.966 \pm 0.017^{*}$ \\
TA-rHDL (apos with neutral TA-rHDL cores) & $0.501 \pm 0.004^{*}$ \\
TA-rHDL (apos with negative-charged TA-rHDL cores) & $0.262 \pm 0.002^{*}$ \\
TA-rHDL ( apos with positive-charged TA-rHDL cores) & $0.816 \pm 0.006^{*}$ \\
TA-rHDL ( denatured apos with neutral TA-rHDL cores) & $0.387 \pm 0.005^{*}$ \\
\hline *means significant differences vs apolipoproteins in free form. & \\
\hline
\end{tabular}




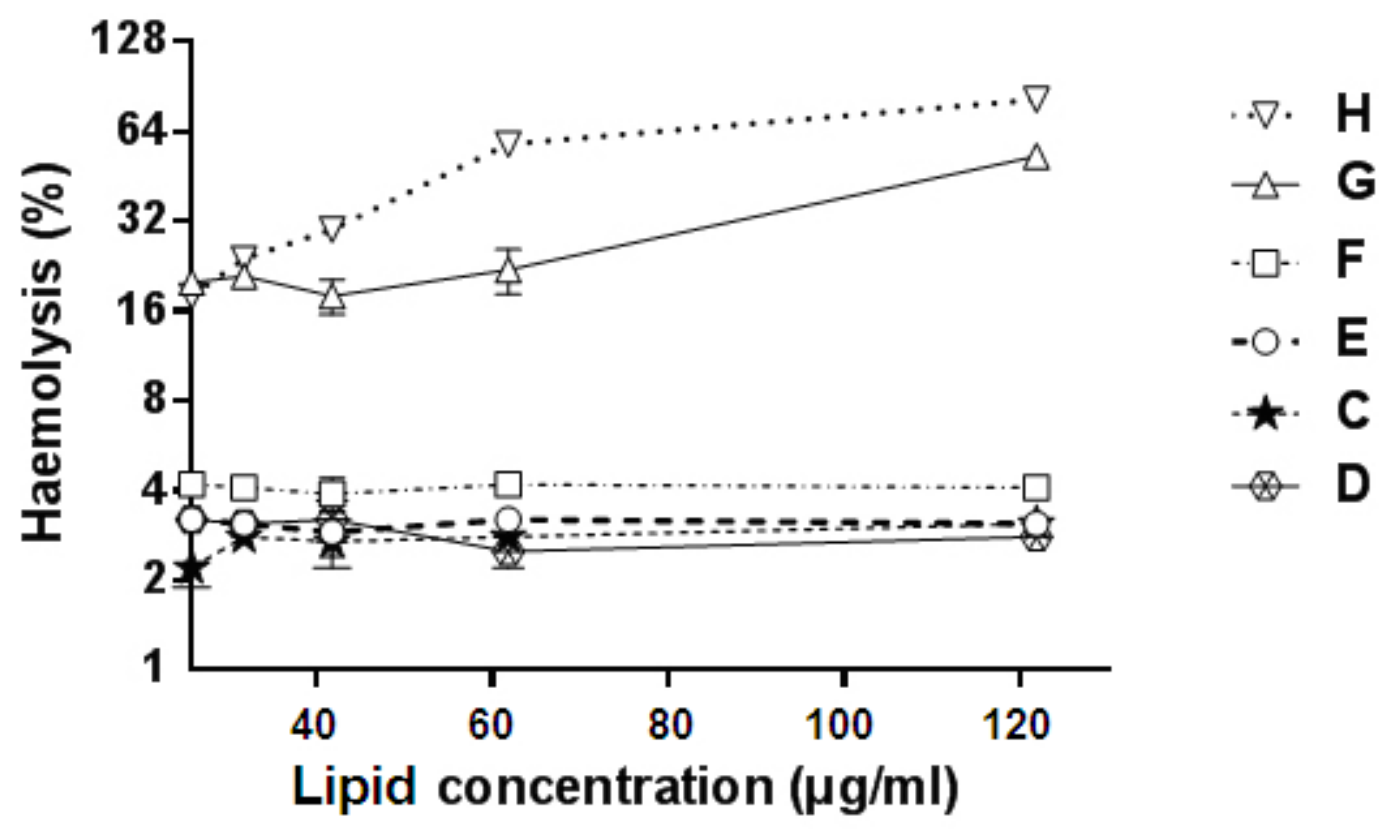

Figure 4. Haemolytic effect of different formulations after incubation with $2 \%(\mathrm{w}: \mathrm{v})$ fresh rabbit red blood cells for $3 \mathrm{~h}$ at $37^{\circ} \mathrm{C}$ (mean $\pm \mathrm{SD}, \mathrm{n}=3$ ). C: blank rHDLs without dialysis; D: blank rHDLs after dialysis; E: TA-rHDLs without dialysis; F: TA-rHDLs after dialysis; G: TA-rHDL cores without dialysis; H: TA-rHDL cores after dialysis.

\section{Sterol efflux from cells}

Cholesterol efflux capacity from macrophages, a metric of HDL function, has a strong inverse association with both carotid intima-media thickness and the likelihood of angiographic coronary artery disease (32). This study was employed to investigate whether the drug-loaded rHDLs with extracted apos still can mediate sterol efflux from macrophages and exert potential antiatherosclerotic effect of the carriers. The results in Figure 6 shows the sterol content left in macrophages after incubation with different formulations. The less sterol content indicates stronger sterol efflux capacity. It was found that TA-rHDLs and TA-rHDL cores (TA liposomes) both led to sterol efflux. The efflux was increased with time and became steady after $24 \mathrm{~h}$. TA-rHDLs with lowest concentration (TA-rHDLl) significantly promoted sterol efflux by $6.16 \%$, which was more than that of TA-rHDL cores (TA liposomes) with high concentration (3.53\%). However, BSA as the negative control and free drug showed no differences between various incubation periods, which suggested no interference on sterol efflux by
BSA or the drug. Figure 6 also shows that the concentration of TA-rHDLs was positively correlated with its sterol efflux capacity, indicating the concentration of TA-rHDLs carriers instead of TA was crucial in mediating sterol efflux. In contrast, the sterol efflux capacity of native HDLs was between those of low and middle concentration of TA-rHDLs. Although higher concentration of TA-rHDLs contained more cholesterol which was included in their formulation, their sterol efflux was higher without being influenced by the slightly higher initial sterol level. It was reported that there were ATP-binding cassette A1 (ABCA1)-mediated (33) and scavenger receptor class $B$ type I (SR-BI)-mediated (34) cholesterol efflux with apo-AI-containing rHDLs. Furthermore, SR-BI-mediated cholesterol efflux is size-dependent in that large rHDLs promote more efflux than small rHDLs (34). The enhanced efflux of large rHDLs is probably due to a combination of greater phospholipid content and more favorable apoA-I conformation (35). Therefore, TA-rHDLs with larger size may mediate cholesterol efflux in the same way. 

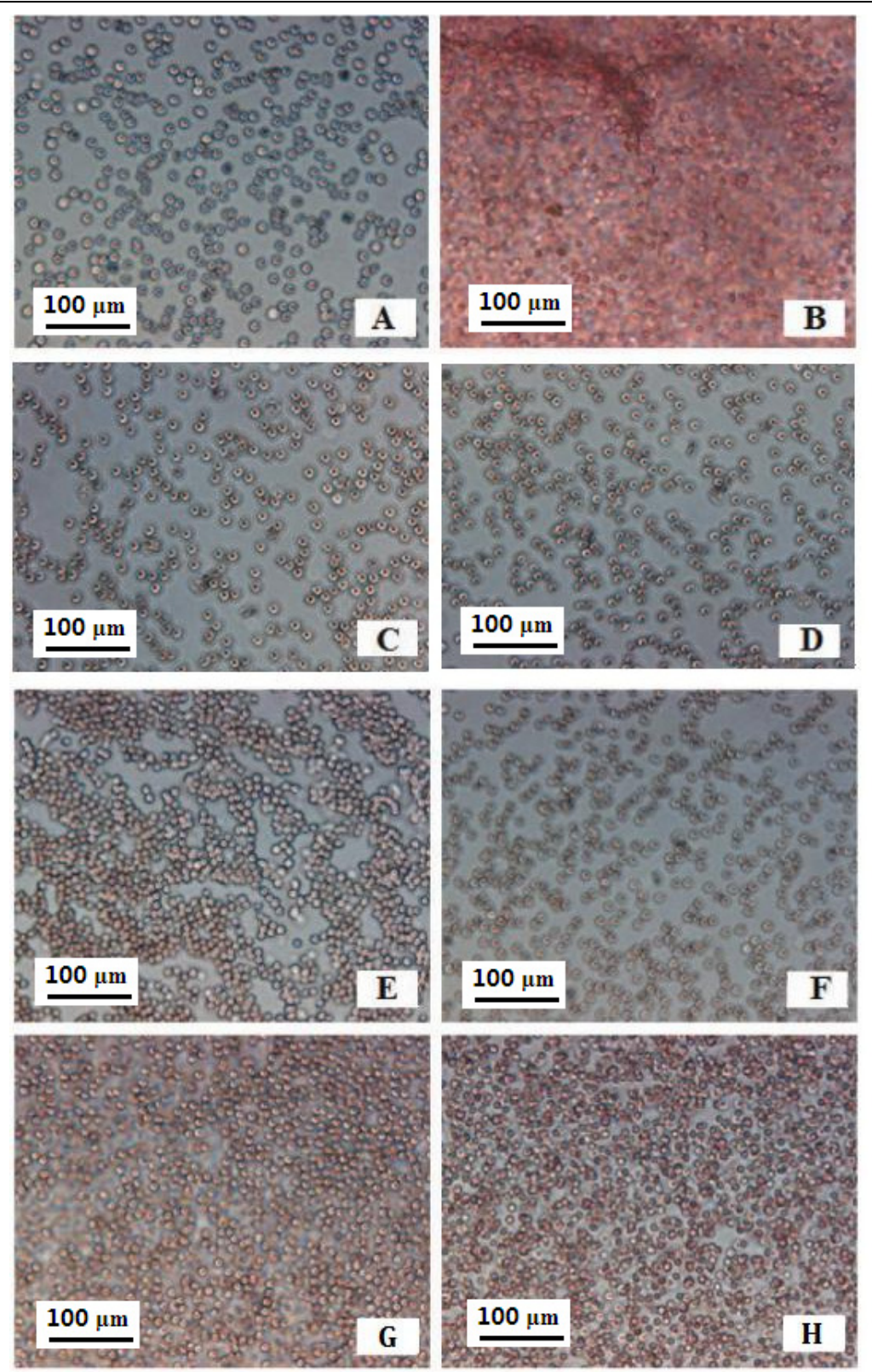

Figure 5. Representative micrographs of 2\% (w:v) rabbit red blood cells incubated with different samples for $3 \mathrm{~h}$ at 37 ${ }^{\circ} \mathrm{C}$. A: normal saline (negative control); B: distilled water (positive control); C: blank rHDLs without dialysis; D: blank rHDLs after dialysis; E: TA-rHDLs without dialysis; F: TA-rHDLs after dialysis; G: TA-rHDL cores without dialysis; H: TA-rHDL cores after dialysis.

\section{Structure remodeling of TA-rHDLs}

Remodeling of the HDLs from the discoidal form to the spherical was important process of reverse cholesterol transport in vivo which was considered as the key protective and therapeutic mechanism of HDLs toward atherosclerosis. Here the remolding process was mimicked by incubating disk-like
TA-rHDLs with LCAT and determined in terms of decreased cholesterol content and microstructure.

As shown in Figure 7 (A), the percentage of free cholesterol decreased most after reacting for 30 min, indicating that 30 min may be the optimal period for structure remolding of rHDLs. 


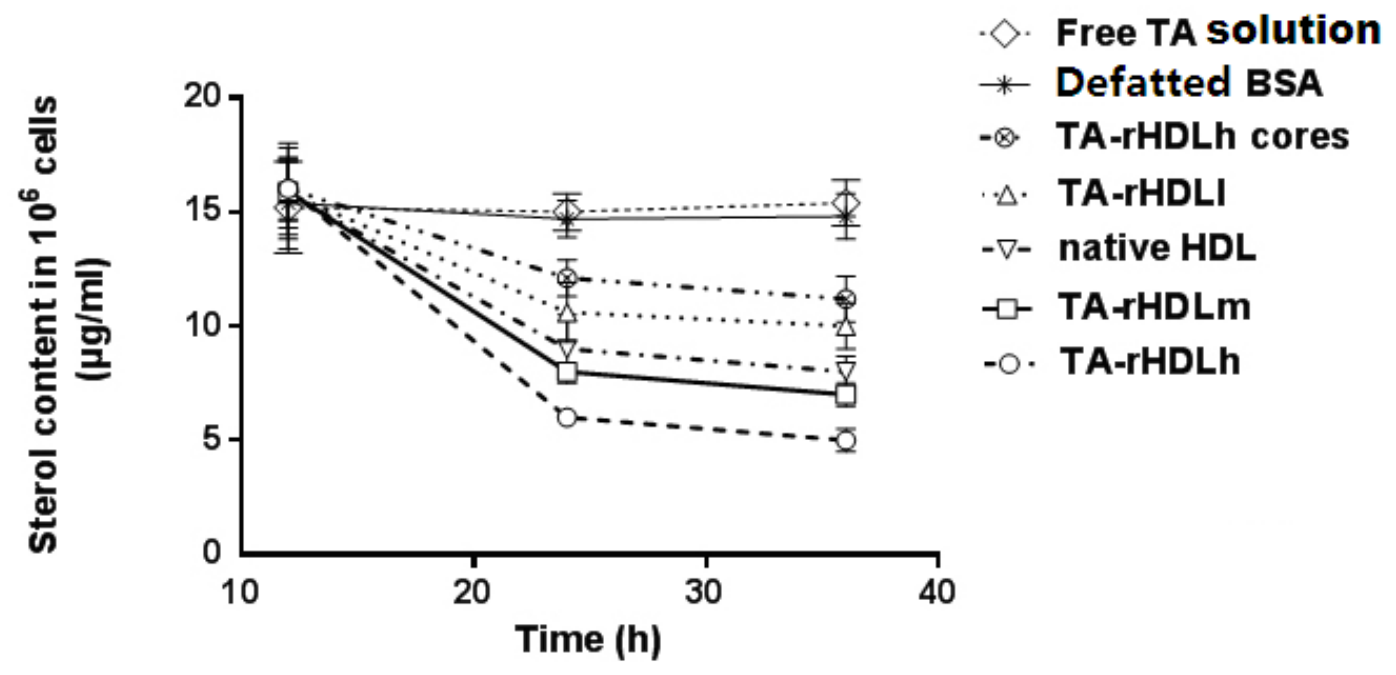

Figure 6. Sterol content in 1000000 macrophages after incubation with different test samples (mean \pm SD, $n=6)$ for 36 $\mathrm{h}$ at $37^{\circ} \mathrm{C}$. TA-rHDLl: low concentration of TA-rHDLs $(1 \mu \mathrm{g} / \mathrm{ml} \mathrm{TA})$; TA-rHDLm: middle concentration of TA-rHDLs $(2 \mu \mathrm{g} / \mathrm{ml}$ TA); TA-rHDLh: high concentration of TA-rHDLs $(4 \mu \mathrm{g} / \mathrm{ml}$ TA); TA-rHDLh cores: high concentration of TA liposomes $(4 \mu \mathrm{g} / \mathrm{ml} \mathrm{TA})$; Free drug solution: $4 \mu \mathrm{g} / \mathrm{ml}$ TA.
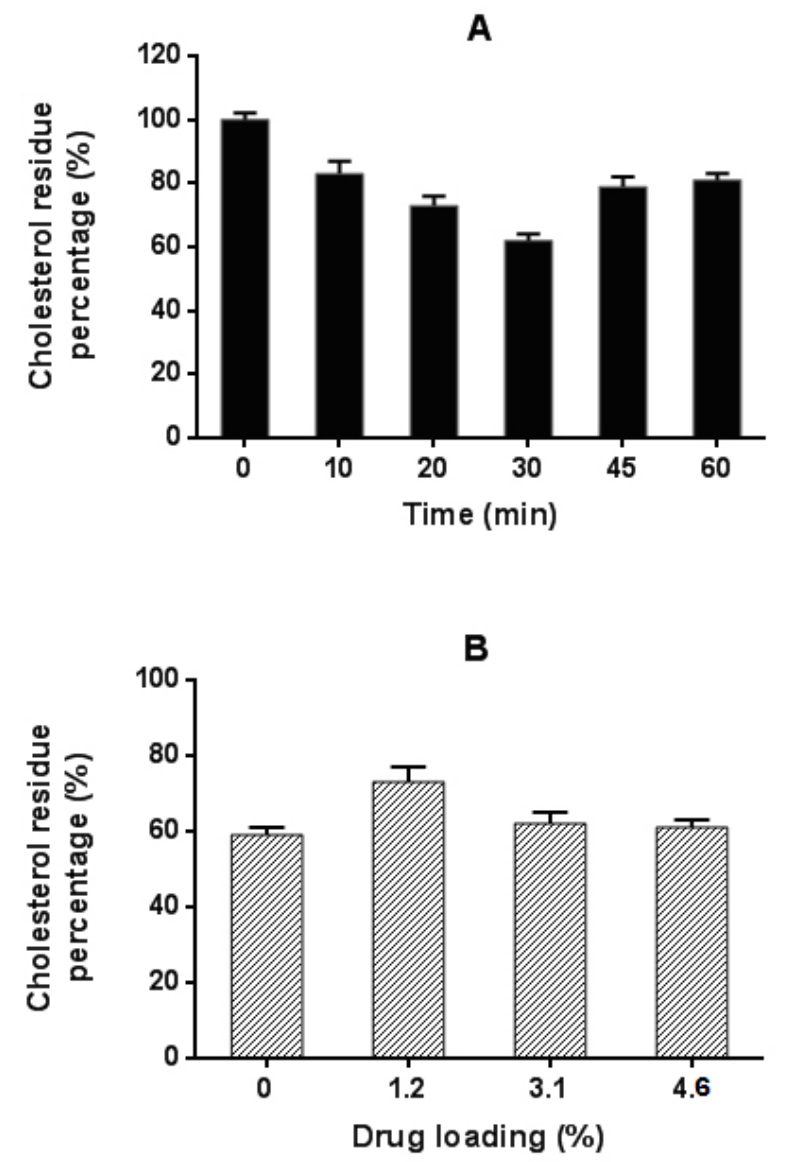

Figure 7. A. Cholesterol residue in TA-rHDLs (drug loading was all kept at 4.6\%) after incubation with LCAT for different time periods at $37^{\circ} \mathrm{C}$ (mean $\pm \mathrm{SD}, \mathrm{n}=3$ ). B. Cholesterol residue in TA-rHDLs with different drug loadings after incubation with LCAT for $30 \mathrm{~min}$ at $37^{\circ} \mathrm{C}($ mean $\pm \mathrm{SD}, \mathrm{n}=3)$. The initial content of cholesterol in TA-rHDLs at " 0 " time point was considered as $100 \%$. 
The concentration of cholesterol residue after 30 min determined by HPLC changed from 0.34 to $0.21 \mu \mathrm{mol} / \mathrm{ml}$, which decreased $37.37 \%$ compared to the initial cholesterol content (seen as $100 \%$ ). However, cholesterol residues were increased after reacting over $30 \mathrm{~min}$. This may be explained by renewed generation of cholesterol from $\mathrm{CE}$ hydrolysis for long periods. Hence, $30 \mathrm{~min}$ were selected as the optimal reaction period. As for the drug loading, compared to blank rHDLs, cholesterol residue percentages were slightly higher with drug loaded rHDLs. However, there were no significant differences $(p>0.05)$ between them and between different drug-loading levels. This indicated few effects of drug on structure remodeling behavior of rHDLs with drug-loading in the range of $1.2 \% \sim 4.6 \%$.

The structure remodeling was also observed by TEM. Seen from Figure 3, TA-rHDLs were predominately discs before reaction (Figure $3 \mathrm{~B}$ ). Then most of them appeared to be round with or without a water cavity (Figure $3 \mathrm{C}, \mathrm{D}$ ) after addition of LCAT for $30 \mathrm{~min}$. From the results of this study, it was evident that the apos extracted from FIV precipitate can activate LCAT to catalyze remolding reaction. Also, TA-rHDL discs with a magnified size could still remold their structure.

\section{CONCLUSIONS}

A simple method was introduced to extract the whole apolipoproteins (apos) in native HDLs from industrial waste. TA-rHDLs prepared using the extractant were demonstrated discs resembling native nascent HDLs, whereas their cores without apos were similar to liposomes. Apos corona in TA-rHDLs increased their structural stability and biocompatibility. TA-rHDLs with enlarged size were testified to be bionic to mediate sterol efflux and remold structure by the action of LCAT, which were key steps for function of reverse cholesterol transportation belonging to native HDLs. Therefore, the extracted apos from FIV precipitate can maintain the biological function of native HDLs. rHDLs could be potential nanocarriers for lipophilic cardiovascular drugs and simultaneously provide dual therapeutic effects of drug and carriers.

\section{ACKNOWLEDGEMENTS}

This study is financially supported by National Science Foundation Grant of China (No. 81273466),
Specialized Research Fund for the Doctoral Program of Higher Education of China (No. 20120096120005) and the Priority Academic Program Development of Jiangsu Higher Education Institutions. We also would like to acknowledge the invaluable assistance of Tonrol Bio-Pharmaceutical Co., Ltd (Anhui, China) by providing raw material for isolation of apolipoproteins.

\section{DECLARATION OF INTEREST}

The authors report no conflicts of interest.

\section{ABBREVIATIONS}

rHDL: reconstituted high density lipoproteins; apos: apolipoproteins;

TA-rHDLs: Tanshinone IIA-loaded reconstituted high density lipoproteins;

LCAT: lecithin:cholesterol acyl transferase;

RCT: reverse cholesterol transportation;

SDS-PAGE: sodium dodecyl sulfate

polyacrylamide gel electrophoresis;

TEM: transmission electron microscopy.

\section{REFERENCES}

1. Viles-Gonzalez JF, Fuster V, Corti R, Badimon JJ. Emerging importance of HDL cholesterol in developing high-risk coronary plaques in acute coronary syndromes. Curr. Opin. Cardiol. 2003;18(4):286-294.

2. Lusis A. Athemsclerosis. Nature. 2000;407(6801):233-241.

3. Assmann G, Nofer JR. Atheroprotective effects of high-density lipoproteins. Medicine. 2003;54(1):321-341.

4. Pownall HJ, Gotto A. Human plasma apolipoproteins in biology and medicine. Structure and Function of Apolipoproteins. 1992:1-32.

5. Linsel-Nitschke P, Tall AR. HDL as a target in the treatment of atherosclerotic cardiovascular disease. Nat. Rev. Drug Discov. 2005;4(3):193-205.

6. Eriksson M, Carlson LA, Miettinen TA, Angelin B. Stimulation of fecal steroid excretion after infusion of recombinant proapolipoprotein AI: potential reverse cholesterol transport in humans. Circulation. 1999;100(6):594-598.

7. Herbert PN, Bernier DN, Cullinane EM, Edelstein L, Kantor MA, Thompson PD. High-density lipoprotein metabolism in runners and sedentary men. JAMA. 1984;252(8):1034-1037.

8. Rensen P, de Vrueh R, Kuiper J, Bijsterbosch M, Biessen E, van Berkel T. Recombinant lipoproteins: lipoprotein-like lipid particles for drug targeting. 
Adv. Drug Deliv. Rev. 2001;47(2-3):251-276.

9. Lou B, Liao XL, Wu MP, Cheng PF, Yin CY, Fei Z. High-density lipoprotein as a potential carrier for delivery of a lipophilic antitumoral drug into hepatoma cells. World J. Gastroenterol. 2005;11(7):954-959.

10. McConathy WJ, Nair MP, Paranjape S, Mooberry L, Lacko AG. Evaluation of synthetic/reconstituted high-density lipoproteins as delivery vehicles for paclitaxel. Anti-cancer drugs. 2008;19(2):183-188.

11. Zhang W-L, Xiao Y, Liu J-P, Wu Z-M, Gu X, Xu Y-M, et al. Structure and remodeling behavior of drug-loaded high density lipoproteins and their atherosclerotic plaque targeting mechanism in foam cell model. Int. J. Pharm. 2011;419(1):314-321.

12. Zhang W-L, Gu X, Bai H, Yang R-H, Dong C-D, Liu J-P. Nanostructured lipid carriers constituted from high-density lipoprotein components for delivery of a lipophilic cardiovascular drug. Int. J. Pharm. 2010;391(1):313-321.

13. Zhang W, He H, Liu J, Wang J, Zhang S, Zhang S, et al. Pharmacokinetics and atherosclerotic lesions targeting effects of tanshinone IIA discoidal and spherical biomimetic high density lipoproteins. Biomaterials. 2013;34(1):306-319.

14. Matz CE, Jonas A. Micellar complexes of human apolipoprotein AI with phosphatidylcholines and cholesterol prepared from cholate-lipid dispersions. J. Biol. Chem. 1982;257(8):4535-4540.

15. Calabresi L, Gomaraschi M, Rossoni G, Franceschini G. Synthetic high density lipoproteins for the treatment of myocardial ischemia/reperfusion injury. Pharmacol. Ther. 2006;111(3):836-854.

16. Vaisar T, Pennathur S, Green PS, Gharib SA, Hoofnagle AN, Cheung MC, et al. Shotgun proteomics implicates protease inhibition and complement activation in the antiinflammatory properties of HDL. J. Clin. Investig. 2007;117(3):746-756.

17. Saito H, Dhanasekaran P, Nguyen D, Deridder E, Holvoet $\mathrm{P}$, Lund-Katz S, et al. |Á-Helix formation is required for high affinity binding of human apolipoprotein AI to lipids. J. Biol. Chem. 2004;279(20):20974-20981.

18. Song X, Fischer P, Chen X, Burton C, Wang J. An apoA-I mimetic peptide facilitates off-loading cholesterol from HDL to liver cells through scavenger receptor BI. Int. J. Biol. Sci. 2009;5(7):637-646.

19. Lerch PG, Förtsch V, Hodler G, Bolli R. Production and Characterization ofa Reconstituted High Density Lipoprotein for Therapeutic Applications. Vox sanguinis. 1996;71(3):155-164.

20. Mallory JB, Kushner PJ, Protter AA, Cofer CL, Appleby VL, Lau K, et al. Expression and characterization of human apolipoprotein $\mathrm{AI}$ in Chinese hamster ovary cells. J. Biol. Chem.
1987;262(9):4241-4247.

21. Pyle LE, Sawyer WH, Fujiwara Y, Mitchell A, Fidge NH. Structural and Functional Properties of Full-Length and Truncated Human Proapolipoprotein AI Expressed in Escherichia coli. Biochemistry. 1996;35(37):12046-12052.

22. Gu X, Zhang W, Liu J, Shaw JP, Shen Y, Xu Y, et al. Preparation and characterization of a lovastatin-loaded protein-free nanostructured lipid carrier resembling high-density lipoprotein and evaluation of its targeting to foam cells. AAPS PharmSciTech. 2011;12(4):1200-1208.

23. Kruger NJ. The Bradford method for protein quantitation. The protein protocols handbook. 1996:15-20.

24. Lehrer S. Solute perturbation of protein fluorescence. Quenching of the tryptophyl fluorescence of model compounds and of lysozyme by iodide ion. Biochemistry. 1971;10(17):3254-3263.

25. Jain SK, Chaurasiya A, Gupta Y, Jain A, Dagur P, Joshi B, et al. Development and characterization of 5-FU bearing ferritin appended solid lipid nanoparticles for tumour targeting. J. Microencapsul. 2008;25(5):289-297.

26. Matz CE, Jonas A. Reaction of human lecithin cholesterol acyltransferase with synthetic micellar complexes of apolipoprotein AI, phosphatidylcholine, and cholesterol. J. Biol. Chem. 1982;257(8):4541-4546.

27. Lu W, Park TG. Protein release from poly (lactic-co-glycolic acid) microspheres: protein stability problems. PDA journal of pharmaceutical science and technology/PDA. J. Pharm. Sci. Tech. 49(1):13-19.

28. Riesz P, Kondo T. Free radical formation induced by ultrasound and its biological implications. Free Radic. Biol. Med. 1992;13(3):247-270.

29. Segrest JP, Garber DW, Brouillette CG, Harvey SC, Anantharamaiah G. The amphipathic $\alpha$ helix: A multifunctional structural motif in plasma apolipoproteins. Adv. Protein Chem. 1994;45:303-369.

30. Jonas A, Wald J, Toohill K, Krul E, Kezdy K. Apolipoprotein AI structure and lipid properties in homogeneous, reconstituted spherical and discoidal high density lipoproteins. J. Biol. Chem. 1990;265(36):22123-22139.

31. Frank PG, Marcel YL. Apolipoprotein AI: structure-function relationships. J. Lipid Res. 2000;41(6):853-872.

32. Khera AV, Cuchel M, de la Llera-Moya M, Rodrigues A, Burke MF, Jafri K, et al. Cholesterol efflux capacity, high-density lipoprotein function, and atherosclerosis. New England J. Med. 2011;364(2):127-135.

33. Favari E, Lee M, Calabresi L, Franceschini G, Zimetti F, Bernini F, et al. Depletion of 
PREbeta-HDL by human chymase impairs ATP-binding cassette transporter A1-but not SR-B1-mediated lipid efflux to HDL. J. Biol. Chem. 2003; 364: 9930-9936.

34. Thuahnai ST, Lund-Katz S, Dhanasekaran P, de la Llera-Moya M, Connelly MA, Williams DL, et al. Scavenger Receptor Class B Type I-mediated cholesteryl ester-selective uptake and efflux of unesterified cholesterol. J. Biol. Chem.
2004;279(13):12448-12455.

35. Yancey $\mathrm{PG}$, de la Llera-Moya M, Swarnakar S, Monzo P, Klein SM, Connelly MA, et al. High density lipoprotein phospholipid composition is a major determinant of the bi-directional flux and net movement of cellular free cholesterol mediated by scavenger receptor BI. J. Biol. Chem. 2000;275(47):36596-36604. 